\title{
Absence of hysteresis at the Mott-Hubbard metal-insulator transition in infinite dimensions
}

\author{
J. Schlipf ${ }^{(1)}$, M. Jarrell ${ }^{(2)}$, P. G. J. van Dongen ${ }^{(1)}$, N. Blümer ${ }^{(1)}$, S. Kehrein ${ }^{(3)}$, Th. Pruschke ${ }^{(4)}$, and D. Vollhardt ${ }^{(1)}$ \\ (1) Theoretische Physik III, Universität Augsburg, 86135 Augsburg, Germany; ${ }^{(2)}$ Department of Physics, University of \\ Cincinnati, Cincinnati, Ohio 45221; ${ }^{(3)}$ Physics Department, Harvard University, Cambridge, MA 02138; ${ }^{(4)}$ Institut für \\ Theoretische Physik, Universität Regensburg, 93040 Regensburg, Germany
}

(February 19, 1999)

\begin{abstract}
The nature of the Mott-Hubbard metal-insulator transition in the infinite-dimensional Hubbard model is investigated by Quantum Monte Carlo simulations down to temperature $T=W / 140$ ( $W=$ bandwidth). Calculating with significantly higher precision than in previous work, we show that the hysteresis below $T_{\mathrm{IPT}} \simeq 0.022 \mathrm{~W}$, reported in earlier studies, disappears. Hence the transition is found to be continuous rather than discontinuous down to at least $T=0.325 T_{\mathrm{IPT}}$. We also study the changes in the density of states across the transition, which illustrate that the Fermi liquid breaks down before the gap opens.
\end{abstract}

PACS numbers: $71.30 .+\mathrm{h}, 71.27 .+\mathrm{a}, 71.28 .+\mathrm{d}$

The explanation of the nature of the Mott-Hubbard metal-insulator transition, i.e., the transition between a paramagnetic metal and a paramagnetic insulator, is one of the classic and fundamental problems in condensed matter physics [1.2]. Metal-insulator transitions of this type are, for example, found in transition metal oxides with partially filled bands near the Fermi level. For such systems band theory typically predicts metallic behavior. The most famous example is $\mathrm{V}_{2} \mathrm{O}_{3}$ doped with Cr 3-5. In particular, in $\left(\mathrm{V}_{0.96} \mathrm{Cr}_{0.04}\right)_{2} \mathrm{O}_{3}$ the metalinsulator transition is first-order below $T_{c} \simeq 380 \mathrm{~K}$ [ $[$ ], with discontinuities in the ratio of the lattice parameters (the two phases being isostructural, however) and in the conductivity, accompanied by hysteresis.

The Mott-Hubbard transition is caused by electronelectron repulsion. The fundamental features of this transition are traditionally expected [5, 1] to be explainable in terms of the half-filled single-band Hubbard model [6 8],

$$
H=-t \sum_{(\mathbf{i j}), \sigma} c_{\mathbf{i} \sigma}^{\dagger} c_{\mathbf{j} \sigma}+U \sum_{\mathbf{i}} n_{\mathbf{i} \uparrow} n_{\mathbf{i} \downarrow},
$$

which describes electrons hopping on a lattice, interacting with each other through on-site Coulomb repulsion.

On the basis of this model the Mott-Hubbard transition was studied intensively over the last 35 years. Important early results were obtained by Hubbard [9] with a Green function decoupling scheme, and by Brinkman and Rice [10] with the Gutzwiller variational method [7], both at $T=0$. Hubbard's approach yields a continuous splitting of the band into a lower and upper Hubbard band, but does not describe quasiparticle features. By contrast, the Gutzwiller-Brinkman-Rice approach concentrates on the low-energy behavior, the transition being monitored by the disappearance of the quasiparticle peak, but does not produce the upper and lower Hubbard bands. A unified approach, treating both the low energy and high energy features on equal footing, has re- cently become possible within the Dynamical Mean-Field Theory (DMFT) [11], which provides the exact solution of the Hubbard model in the limit of infinite dimensionality (or coordination number) 12]. The complicated structure of the self-consistent DMFT-equations makes an analytic solution untractable and hence one has to resort to approximate techniques. In the last few years Georges, Kotliar and collaborators performed detailed investigations of the metal-insulator transition scenario within the DMFT, by employing iterated perturbation theory (IPT), exact diagonalization (ED) of small systems, quantum Monte-Carlo (QMC) simulations and, at $T=0$, a projective self-consistent technique (PSCT) [11,13]. While the overall transition scenario reported by these authors indeed combines essential features of the early approaches, they find the transition to be discontinuous, with hysteresis, due to a coexistence regime between the metallic and the insulating phase. For all finite temperatures $T<T_{\mathrm{IPT}}$ the quasiparticle weight disappears abruptly and the gap between the Hubbard bands opens discontinuously as a function of $U$. Hence these authors argued that the experimentally observed metal-insulator transition in $\mathrm{V}_{2} \mathrm{O}_{3}$ can already be understood using a purely electronic correlation model.

At $T=0$, numerical renormalization group (NRG) studies [14] also found hysteresis and a value for the critical interaction of $U_{c}=5.86$ (in our units, see below), which agrees with the results of the PSCT [13]. Nevertheless, the existence of a preformed gap at $T=0$ and the corresponding separation of energy scales on which the PSCT is based were recently disputed by one of us [1.5]. Finally, a continuous transition with a considerably lower $U_{c}$ was recently observed within the random dispersion approximation (RDA [2,16]). Clearly the Mott-Hubbard transition scenario is still very controversial.

It is the purpose of this paper to carefully reexamine the nature of the metal-insulator transition within DMFT at finite temperatures. The examination 
of a transition region requires a technique with sufficient precision. Since IPT is a rather ad hoc approximation scheme, its qualitative and quantitative accuracy is uncertain. On the other hand, for $T>0 \mathrm{ED}$ is limited to quite small systems ( $\leq 7$ sites), so that finite-size effects may be considerable. Indeed, although both techniques predict the metal-insulator transition to be discontinuous, their quantitative predictions differ substantially (see below). In fact, their respective regions of hysteresis do not even overlap much. To resolve these discrepancies we perform finite-temperature QMC calculations, using two different codes to reduce possible systematic errors. Although QMC is limited to not too low $T$ - and not too large $U$-values, it is still the best understood and most thoroughly tested technique presently available for the solution of the DMFT-equations. For comparison with Ref. [11 we focus on the Hubbard model with a semi-elliptical non-interacting density of states (DOS): $N(E)=\sqrt{4-\left(E / t^{*}\right)^{2}} /\left(2 \pi t^{*}\right)$ if $|E| \leq 2 t^{*}$ and zero elsewhere. This DOS is realized, e.g., on a Bethe lattice with hopping amplitude scaled as $t=t^{*} / \sqrt{Z}$, where $Z \rightarrow \infty$ is the coordination number. In the following we set $t^{*}=1$. In order to study the Mott-Hubbard transition, we restrict our calculations to the paramagnetic phase and exclude symmetry breaking. The solution of the Hubbard model is then determined by the following single-site effective action $11,17,20$ :

$$
\begin{aligned}
S_{\text {eff }} & =-\int_{0}^{\beta} d \tau d \tau^{\prime} \sum_{\sigma} c_{\sigma}^{\dagger}(\tau) \mathcal{G}_{0}^{-1}\left(\tau-\tau^{\prime}\right) c_{\sigma}\left(\tau^{\prime}\right) \\
& +U \int_{0}^{\beta} d \tau\left(c_{\uparrow}^{\dagger}(\tau) c_{\uparrow}(\tau)-\frac{1}{2}\right)\left(c_{\downarrow}^{\dagger}(\tau) c_{\downarrow}(\tau)-\frac{1}{2}\right) .
\end{aligned}
$$

In particular, the on-site Green function $G_{\mathbf{i i}}\left(\tau-\tau^{\prime}\right)$ of the Hubbard model is identical to the single-site Green function $G\left(\tau-\tau^{\prime}\right) \equiv-\left\langle\mathcal{T} c(\tau) c^{\dagger}\left(\tau^{\prime}\right)\right\rangle_{S_{\text {eff }}}$, which is implicitly determined by $S_{\text {eff }}$ and the self-consistency relation $\mathcal{G}_{0}\left(i \omega_{n}\right)=\left[i \omega_{n}+\mu-G\left(i \omega_{n}\right)\right]^{-1}$, where $\omega_{n}=(2 n+1) \pi T$. The key point in DMFT is thus the accurate calculation of the single-site Green function $G(\tau)$. For this purpose we use QMC-simulations, which are essentially exact though computationally expensive [19,21. After discretizing the imaginary time into $\Lambda$ time slices of length $\Delta \tau=\beta / \Lambda$ and performing a Hubbard-Stratonovich transformation which introduces auxiliary Ising spins, the DMFT-equations are solved by iteration. The number of proposed flips of Ising spins ("sweeps") per iteration will be important here. Each iteration has as input the "old" self-energy $\Sigma_{\text {old }}(i \omega)$ and as output a "new" self-energy $\Sigma_{\text {new }}(i \omega)$. The rate of change in the iteration procedure is measured by $\eta \equiv \Lambda^{-1} \sum_{n} \mid \Sigma_{\text {old }}\left(i \omega_{n}\right)-$ $\Sigma_{\text {new }}\left(i \omega_{n}\right) \mid$. Experience shows that, for most purposes (e.g., calculation of thermodynamic quantities outside the critical regime), convergence is reached if $\eta \leq 10^{-3}$. Physical properties are finally obtained by extrapolation

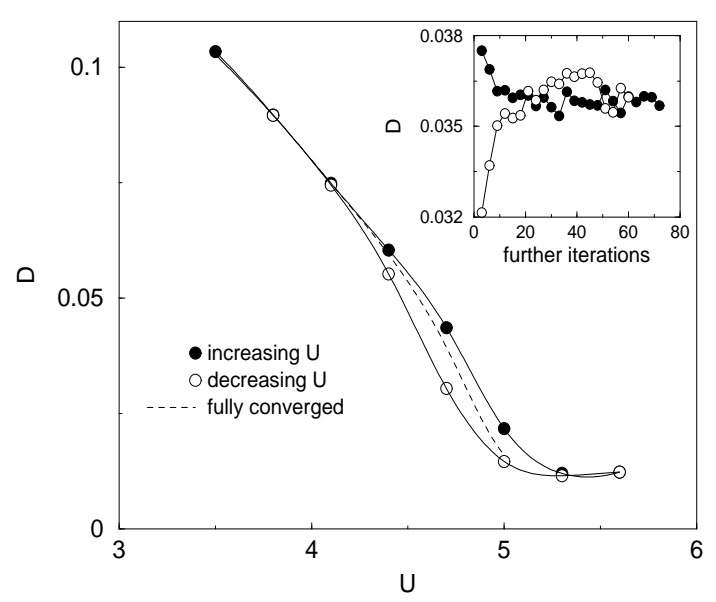

FIG. 1. QMC-results for the double occupancy $D$ at $T=1 / 20$ using the criterion $\eta \leq 10^{-3}$. The error bars are smaller than the data points. All lines are guides to the eye only. Inset: Vanishing of the hysteresis as a function of the number of further iterations at $T=0.05, U=5$ and $\Delta \tau=0.3$.

of the simulation results for various $\Delta \tau$-values to the limit $\Delta \tau \rightarrow 0$.

We found that straightforward implementation of the QMC algorithm with $\eta \leq 10^{-3}$ indeed leads to the apparent convergence of two solutions, i.e., to hysteresis. As an example we present results for the double occupancy $D=\left\langle n_{\mathbf{i} \uparrow} n_{\mathbf{i} \downarrow}\right\rangle$ in Fig. 1. The upper curve (solid dots) shows results for increasing coupling strength, where we use the self-energy calculated for interaction $U$ as input for the calculation for $U+\Delta U$ (here $\Delta U=0.3$ ). Similarly, the lower curve (open dots) shows results for decreasing $U$. At each $U$-value the iteration procedure was terminated as soon as the condition $\eta \leq 10^{-3}$ was satisfied, which typically happens after only a few iterations. In this way we are able to qualitatively reproduce the hysteresis found in Ref. 11] by means of a standard QMC-algorithm with a standard convergence criterion. However, as will be shown below, in the case of the Mott-Hubbard transition the criterion $\eta \leq 10^{-3}$ is not sufficient to guarantee convergence to the true solution.

For this we investigate the stability of the two solutions independently under further iterations. As an example we consider the solution at $T=1 / 20$ and $U=5$. The data for $\Delta \tau=0.3$ are presented in the inset of Fig. 1. In order to obtain maximum accuracy we use a large number of sweeps per iteration $\left(2-3 \cdot 10^{5}\right)$. We find that $(i)$ both solutions at $U=5$ in Fig. 1 are unstable, (ii) a new stable solution is reached after approximately 20 more iterations, and (iii) the solution with the larger number of sweeps and time slices (solid dots) reaches equilibrium sooner and has smaller fluctuations. During the iteration process $\eta$ fluctuates around $\langle\eta\rangle \simeq 4 \cdot 10^{-4}$ and gives essentially no information about the distance from equilibrium. We obtained similar results for a dense grid of 


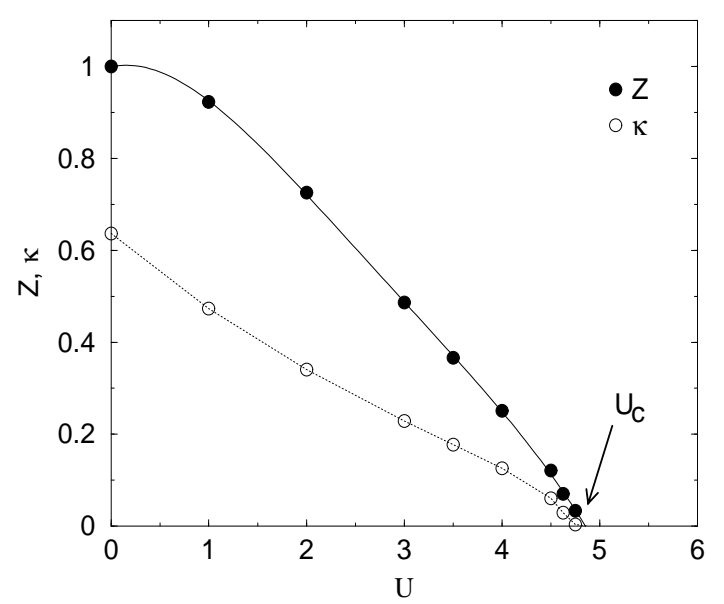

FIG. 2. Compressibility $\kappa$ and quasiparticle renormalization factor $Z=m / m^{*}$ vs. $U$ for $T=1 / 15$. All lines are guides to the eye only.

other values for $U$ and $\Delta \tau$. Extrapolating to $\Delta \tau \rightarrow 0$ and combining the results for various $U$, we obtain a smooth curve $D(U)$ without hysteresis (dashed curve in Fig. 1). The same happens at higher temperatures $(T=1 / 10$, $1 / 13$ and $1 / 15)$ and also at lower temperatures, such as $T=1 / 30$ and $T=1 / 35$.

We also studied the quasiparticle renormalization factor $Z=m / m^{*}$ and the compressibility $\kappa$ at various temperatures. After 15-25 further iterations, none of these quantities shows hysteresis any more. The results for $T=1 / 15$ are shown in Fig. 2. We locate the MottHubbard transition at the interaction strength $U_{c}$ where $Z(U)$ and $\kappa(U)$ essentially vanish. The resulting phase diagram is plotted in Fig. 3, where the corresponding IPT- and ED-results 11,22 and the $U_{c}$-values at $T=0$ obtained by PSCT [13], NRG 14] and RDA [16] are also shown. There is a clear quantitative and qualitative discrepancy between our numerically exact QMC-data and both the ED- and the IPT-results. According to IPT, the transition below $T=T_{\mathrm{IPT}} \simeq 0.088$ is discontinuous. By contrast, we find that the transition from the metal to the insulator is continuous down to at least $T=1 / 35 \simeq 0.325 T_{\mathrm{IPT}}$.

More information concerning the Mott-Hubbard transition can be obtained from the DOS, which we calculated from the converged data for the Green function using the Maximum Entropy Method (MEM [23). Our results for the DOS as a function of $U$ at $T=1 / 20$ are presented in Fig. 4. Upon increase of $U$, the DOS develops a welldefined central peak and shoulders, with the peak pinned at its Fermi liquid value. The peak rapidly collapses at $U \simeq U_{c}$ (here: $U_{c} \simeq 4.8$ ), leaving an incomplete gap [24]. Beyond $U_{c}$ the system remains semi-metallic until at $U_{g}>U_{c}$ (here: $U_{g} \simeq 5.0$ ) an actual gap opens (within numerical accuracy). In order to better under-

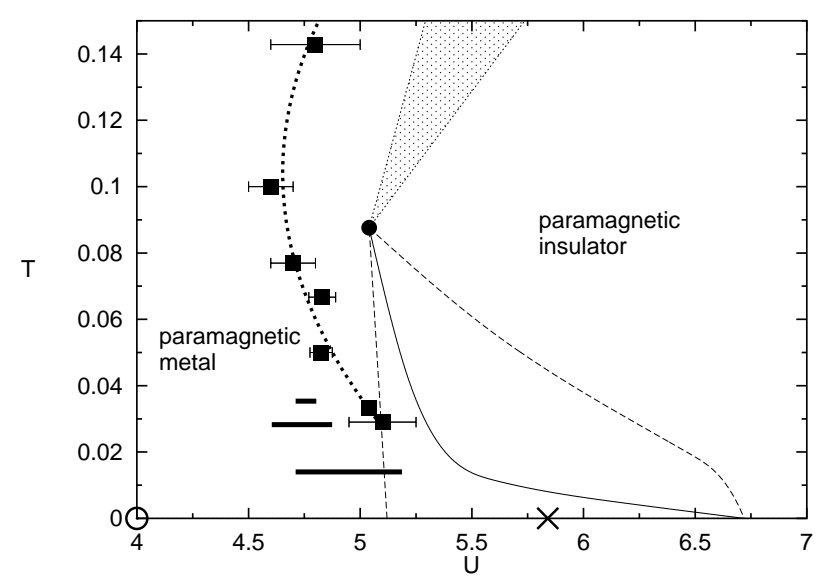

FIG. 3. Phase diagram of the Hubbard model (paramagnetic phase only). Solid squares: continuous metal-insulator transition calculated with QMC (error bars include both the statistical errors and the uncertainty in $U_{c}$ due to finite temperatures). The dotted line is a guide to the eye only. Broad horizontal lines: coexistence region within ED [1. 22]. Dashed lines: coexistence region within IPT; the line of first-order transitions (full curve) ends at $T_{\text {IPT }}$ (solid circle) 11]. The shaded area is a crossover region. Also shown are the $U_{c}$-values from PSCT/NRG $(\mathrm{X})$ and RDA (O).

stand the region $U_{c}<U<U_{g}$ we studied the temperature dependence of the screened local moment (inset to Fig. 4) and of the spectrum (not shown) at $U=4.8$, starting from $T=1 / 20$. Upon increase of $T$ the incomplete gap and the screened local moment remain essentially unchanged. Upon decrease of $T$ the central peak and the Fermi-liquid behavior are rapidly restored, and the screened moment falls dramatically. The incomplete gap and the temperature-independence of the screened moment imply that for $U_{c}<U<U_{g}$ there are few electronic states at the Fermi energy to screen the spins. The behavior in this region can be interpreted as emanating from a quantum critical point at $T=0$, characterized by a vanishing DOS at the Fermi level, $N(0)=0$. The depletion of screening states obtains a natural interpretation in terms of Nozières' "exhaustion" scenario [25], which was recently found to be realized in the periodic Anderson model [26].

To clarify the influence of band-structure effects we also performed calculations for a hypercubic lattice in $d=\infty$ including next-nearest-neighbor hopping amplitudes $t^{\prime}=t^{\prime *} / \sqrt{2 d(d-1)}$ [27,28]. Choosing $t^{\prime *} / t^{*}<0$ in order to obtain a finite lower band edge, we find the hysteresis effects to be strongly suppressed by frustration, e.g., no initial hysteresis was observed for $t^{\prime *} / t^{*} \lesssim-0.25$. The phase diagram for $t^{\prime}=0$, previously obtained 229] using $\mathrm{QMC}$ and perturbation theory in $t^{*}(\mathrm{NCA})$, is qualitatively similar to that for the Bethe lattice in Fig. 3.

In summary, we demonstrated that, for temperatures 


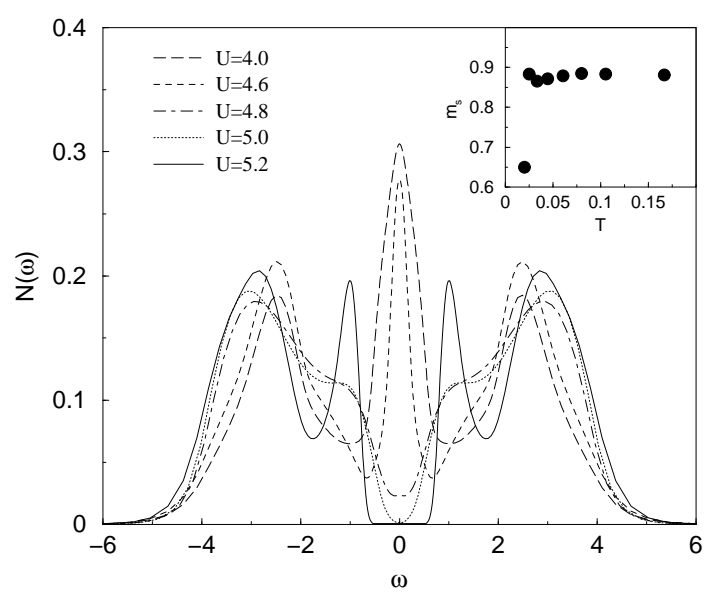

FIG. 4. QMC/MEM-results for the DOS (Bethe lattice) for various values of $U$ at $T=1 / 20$, and $\Delta \tau=0.017$. The gap opens at $U_{g} \simeq 5.0$. The temperature-dependence of the screened local moment $m_{s}$ (the product of the temperature and the local susceptibility) is shown in the inset for $U=4.8$.

down to $T_{\min }=1 / 35 \simeq 0.325 T_{\mathrm{IPT}}$, the coexistence region characteristic of a first-order metal-insulator transition disappears in sufficiently careful QMC simulations. Further conclusions about the order of the transition cannot safely be made. Physically we would expect the transition to be a smooth (broadened by finite temperatures) but rapid crossover between $U_{c}$ (where the Fermi liquid breaks down) and $U_{g}>U_{c}$ (where the gap opens). We cannot rule out the existence of a first-order transition at even lower temperatures and note that our results may be smoothly connected to those from PSCT [13] and NRG 114] at $T=0$. However, we found no evidence for the existence of a preformed gap at low temperatures, in line with the transition scenarios of Refs. [2,15,25]. - For a bandwidth of $0.8 \mathrm{eV}$ [30] our results imply that down to $T_{\min } \simeq 70 \mathrm{~K}$ the metal-insulator transition is continuous. Since in the experiment on Cr-doped $\mathrm{V}_{2} \mathrm{O}_{3}$ the transition is first-order below $T_{c} \simeq 380 \mathrm{~K}$ [1], an explanation of the nature of this transition apparently requires the inclusion of other degrees of freedom, such as lattice [31], orbital [32,33 and possibly higher-spin [34] effects.

We are grateful to R. Bulla, F. Gebhard, K. Held, W. Hofstetter, S. Horn, R. Noack, G. Kotliar, H.-R. Krishnamurthy, W. Krauth, M. Rozenberg, M. Ulmke, and F. C. Zhang for discussions. Support by NSF grants DMR-9704021 and DMR-9357199 and the Ohio Supercomputer Center (MJ) and by the John von Neumann Institute for Computing, Jülich, (JS, NB, DV) is acknowledged.
[1] N. F. Mott, Rev. Mod. Phys. 40, 677 (1968); MetalInsulator Transitions (Taylor \& Francis, London, 1990).

[2] F. Gebhard, The Mott Metal-Insulator Transition (Springer, Berlin, 1997).

[3] D. B. McWhan and J. P. Remeika, Phys. Rev. B2, 3734 (1970).

[4] D. B. McWhan et al., Phys. Rev. B7, 1920 (1973).

[5] T. M. Rice and D. B. McWhan, IBM J. Res. Develop. 251 (May 1970).

[6] J. Hubbard, Proc. Roy. Soc. London A276, 238 (1963).

[7] M. C. Gutzwiller, Phys. Rev. Lett. 10, 59 (1963).

[8] J. Kanamori, Prog. Theor. Phys. 30, 275 (1963).

[9] J. Hubbard, Proc. Roy. Soc. London A281, 401 (1964).

[10] W. F. Brinkman and T. M. Rice, Phys. Rev. B2, 4302 (1970).

[11] For a review, see A. Georges, G. Kotliar, W. Krauth and M. J. Rozenberg, Rev. Mod. Phys. 68, 13 (1996).

[12] W. Metzner and D. Vollhardt, Phys. Rev. Lett. 62, 324 (1989).

[13] G. Moeller et al., Phys. Rev. Lett. 74, 2082 (1995).

[14] R. Bulla, Th. Pruschke and A. C. Hewson, J. of Phys.: Condens. Matter 10, 8365 (1998); R. Bulla, cond-mat preprint (1999).

[15] S. Kehrein, Phys. Rev. Lett. 81, 3912 (1998).

[16] R. Noack and F. Gebhard, cond-mat preprint 9810222.

[17] U. Brandt and C. Mielsch, Z. Phys. B75, 365 (1989).

[18] V. Janiš, Z. Phys. B83, 227 (1991).

[19] M. Jarrell, Phys. Rev. Lett. 69, 168 (1992); M. Jarrell, H. Akhlaghpour, and Th. Pruschke, in Quantum Monte Carlo Methods in Condensed Matter Physics, Ed. M. Suzuki (World Scientific, 1993), pp. 221-34.

[20] A. Georges and G. Kotliar, Phys. Rev. B45, 6479 (1992).

[21] M. Ulmke, V. Janiš and D. Vollhardt, Phys. Rev. B51, 10411 (1995).

[22] W. Hofstetter, private communication.

[23] M. Jarrell and J. E. Gubernatis, Physics Reports 269, 133 (1996).

[24] The breakdown of the Fermi liquid does not coincide with the transition to the insulator; similar behavior is found for normal liquid ${ }^{3} \mathrm{He}$ upon increasing pressure.

[25] P. Nozières, Eur. Phys. J. B6, 447 (1998); D. E. Logan and P. Nozières, Phil. Trans. R. Soc. London A356, 249 (1998).

[26] A. N. Tahvildar-Zadeh, M. Jarrell and J. K. Freericks, Phys. Rev. Lett. 80, 5168 (1998).

[27] J. Schlipf, doctoral thesis, Univ. Augsburg (1998).

[28] E. Müller-Hartmann, Z. Phys. B74, 507 (1989).

[29] M. Jarrell and Th. Pruschke, Z. Phys. 90, 187 (1993).

[30] This value was argued in Ref. [11], p. 81, to be close to the experimental bandwidth.

[31] P. Majumdar and H. R. Krishnamurthy, Phys. Rev. Lett. 73, 1525 (1994).

[32] C. Castellani, C. R. Natoli and J. Ranninger, Phys. Rev. B18, 4945 (1978).

[33] W. Bao et al., Phys. Rev. Lett. 78, 507 (1997).

[34] S. Yu. Ezhov et al., cond-mat preprint 9901229. 